Pensamiento Crítico Vol. 19 № 2, pp. 151-156

\title{
La teoría de funciones: La matemática frente a la economía
}

Function theory: mathematics and economics

Luis Mejía M. ${ }^{1}$

\section{RESUMEN}

En la vida diaria continuamente surgen situaciones en las cuales dos o más magnitudes se relacionan entre sí mediante alguna regla. Es ahí donde, sin darnos cuenta, están presentes las funciones, y lo más sorprendente es que, aun sin conocerlos formalmente, ilas estamos utilizando!

Reconocer que en determinada situación está presa una función y poder establecer un modelo económico que la represente es de gran utilidad, ya que con una ecuación podemos realizar un análisis de la situación y hacer predicciones.

Palabras clave: Relación, correspondencia, regla, dominio, predicción, teoría.

\begin{abstract}
In the daily life constant situations arise in which two or more magnitudes relate between yes by means of some rule. It is there where, without realizing, the functions are proposed, and the most surprising thing is that still without knowing them formally we are using them!.
\end{abstract}

1 Matemático economista. 


\section{Pensamiento Crítico Vol. 19. No 2}

To admit that in certain situation a function is imprisoned yes and to be able to establish an economic model who represents her is of great usefulness since with an equation we can realize an analysis of the situation and do predictions.

Keywords: Relation, correspondence, rule, domain, prediction, theory.

La creciente contribución de la matemática a la cultura del mundo moderno y su importancia como parte vital de la formación matemática del economista han hecho necesario que la matemática en la facultad de Economía se seleccione juiciosamente y que se enseñe bien.

Uno de los prerrequisitos para el mejoramiento de la enseñanza de la matemática en la facultad de economía es un mejor programa de estudios, un programa que tome en consideración el uso creciente de la matemática en economía y que, a la vez, refleja los avances recientes de la matemática en economía.

De toda la matemática que se estudia en economía, ninguna es más importante que la teoría de funciones, en parte porque puede servir de base a la construcción de modelos económicos, pero sobre todo por ser una de las ideas fundamentales en matemática. Casi cualquier estudio que se refiere a la aplicación de la matemática a problemas prácticos o que requieran el análisis de datos empíricos emplea este concepto matemático.

Para saber de qué manera ayudan la matemática a la economía, revisemos algunos de los fines que el estudio de la economía espera alcanzar.

Una de las tareas de la economía consiste en describir y resumir las relaciones complejas que ocurren en el comportamiento económico de los hombres y de las naciones. Otra de las tareas consiste en formular proposiciones sobre las relaciones de comportamiento, a fin de hacer predicciones y recomendaciones sobre política. Estas proposiciones están basadas en:

1. La descripción y el resumen de lo que se observa en la realidad.

2. Supuestos comprobados o no comprobados que establecemos sobre las motivaciones y medios ambientes de las unidades de comportamiento. El método y las herramientas matemáticas son de gran ayuda para el desempeño de esas teorías.

\section{2}




\section{Luis Mejía M.}

Nuestra atención se centra ahora en esta importante herramienta matemática. Con el fin de adquirir una comprensión práctica de las múltiples formas de utilizar la teoría de funciones.

Una función expresa la idea de que una cantidad depende o está determinada por otra. Los siguientes ejemplos aclaran la idea:

- El costo de publicar en un periódico un aviso de venta de una vivienda depende del número de palabras que tiene el texto publicado.

- El valor de las unidades de inversión depende del tiempo (días) ya que están cambiando diariamente.

- El costo del servicio telefónico depende de la cantidad de llamadas adicionales realizadas durante el mes.

- Las prestaciones otorgadas por el sistema de seguridad social de un país depende de su tasa de desempleo.

- El poder adquisitivo de la moneda depende del índice del costo de la vida.

- La cantidad de cierto artículo que el fabricante ofrecerá depende del precio que puede lograr.

Si analizamos las situaciones anteriores nos daremos cuenta de que en todas estas cosas existe una relación entre dos magnitudes y que una de las magnitudes depende de la otra; en matemática, para describir esa relación usamos el concepto de función. Si una función se expresa por una relación del tipo

$$
Y=f(x)
$$

Entonces $\mathrm{X}$ se denomina la variable independiente o argumento de $f$ e $y$ se conoce como la variable dependiente. 


\section{Pensamiento Crítico Vol. 19. No 2}

En general, encontramos funciones que se expresan estableciendo el valor de la función por medio de una fórmula algebraica en términos de la variable independiente de que se trate.

Los ejemplos dados anteriormente demuestran la importancia de las funciones, como medio de representación de relaciones económicas en general, se usan frecuentemente funciones para representar diversas formas de conducta. Por ejemplo, se puede utilizar funciones para representar una conducta humana (tal como la oferta de horas de trabajo a varias tarifas salariales) o proceder técnico (tal como la relación entre la producción física de un bien y el insumo de trabajo usado para esa tal producción). Se debe notar que esa función puede aparecer siempre en forma general como

$$
Q_{d}=f(p)
$$

O en forma específica como

$$
Q_{d}=f(p)=10-2 P
$$

Reconocer que en determinada situación está presente una función y poder establecer un modelo económico que la represente es de gran utilidad ya que con una fórmula podemos realizar un análisis de la situación y hacer predicciones.

Tanto en economía como en administración, y en general cualquier estudio realizado con un método científico, interesa precisar las relaciones que existen entre los diversos entes bajo observación. Como toda ciencia se expresa a través de un lenguaje o símbolo, la principal diferencia entre las funciones matemáticas y las funciones en economía reside en que en la primera los supuestos y conclusiones se formulan utilizando símbolos matemáticos y no palabras, ecuaciones y no proposiciones. Dado que los símbolos y las palabras son realmente equivalentes (como lo demuestra el hecho de que aquellos suelen definirse con palabras), poco importa que se utilicen unos u otros, pero es quizás indiscutible que los símbolos resultan más convenientes para el razonamiento deductivo y que, sin duda, permiten formular enunciados más concisos y exactos; pero la matemática tiene la ventaja de obligar al analista a exponer en forma explícita sus supuestos en todas las etapas del razonamiento. Otra de las ventajas más importantes se 


\section{Luis Mejía M.}

derivan de la utilización de la matemática en la investigación científica de la economía, tanto respecto a la precisión y simplificación del método de trabajo como en cuanto a la posibilidad de ampliar notablemente el campo de conocimiento económico, obliga a los economistas actuales a disponer de una adecuada formación matemática.

Las relaciones que pueden establecerse entre ciertas magnitudes variables como son precios, costos, tasa de interés, etc. Se pueden presentar mediante funciones numéricas, aunque la generación y el significado de estas funciones deben puntualizarse mucho desde el punto de vista económico antes de manejarlas como meros entes matemáticos.

Cuando la relación entre un conjunto de variables económicas puede ser expresada por una ley matemática, se obtiene lo que se llama una relación funcional que facilita notablemente el análisis de los problemas económicos en los que intervienen las variables. Sin embargo, en general es prácticamente imposible obtener una ley matemática rígida que describa en forma exacta un fenómeno económico, porque hay variables económicas que son muy difíciles de medir y hay que conformarse con estimaciones que en muchos casos son discutibles. Por otra parte, hay conceptos económicos que no pueden ser precisados de manera específica como el concepto de "utilidad" o satisfacción. En consecuencia es importante tener plena conciencia de las limitaciones que tiene las expresiones matemáticas de fenómenos económicos.

Esperamos sinceramente que este artículo señale el camino para precisar el "quantum" de conocimientos matemáticos que necesita el economista. La revisión de los planes de estudio de la economía en la facultad. La teoría de funciones proporcionará los elementos básicos para la modelación de la faceta matemática del economista. También debe considerarse como una muestra de la clase de programa de estudios y como una fuente de sugerencias para los profesores de la Facultad de Ciencias Económicas.

\section{Referencias bibliográficas}

Alcalde, Ángel (1992). Cálculo infinitesimal para economistas. Ed. Aguilar.

Berenson D.M. Leonor (1998). Estadística para administración y economía. McGraw Hill. 


\section{Pensamiento Crítico Vol. 19. № 2}

Chiang, A (1984). Métodos fundamentales de economía matemática. Edit. McGraw Hill.

Galván Sánchez, Delia Aurora y otros (2006). Cálculo diferencial para administración y ciencias sociales. Ed. Pearson Educación de México.

García Gonzales, Víctor (1991). Econometría para la planificación. G. Herrera Editores.

Monserrat Díaz Fernández, María del Mar; Llorente M. (1991). Economía. 3era Edic. Ediciones Pirámide. 